\title{
Z3 Research Square \\ Preoperative factors predicting the preservation of the posterior cruciate ligament in total knee arthroplasty
}

\section{Qiang Liu ( $\square$ liuqiang_pku@126.com )}

Peking University People's Hospital, Peking University

\section{Yi Wang}

Beijing Huairou Hospital of Beijing Traditional Chinese Medicine

\section{Liyi Zhang}

Peking University People's Hospital, Peking University

Jianhao lin

Peking University People's Hospital, Peking University

\section{Diange Zhou}

Peking University People's Hospital, Peking University

\section{Research Article}

Keywords: Knee, Total knee arthroplasty, PCL retaining, PCL substituting, Risk factor

Posted Date: February 14th, 2022

DOI: https://doi.org/10.21203/rs.3.rs-1344655/v1

License: (c) (i) This work is licensed under a Creative Commons Attribution 4.0 International License. Read Full License 


\section{Abstract}

Objective: We aimed to analyze the preoperative factors predicting the successful preservation of the posterior cruciate ligament (PCL) in total knee arthroplasty (TKA).

Methods: In this retrospective study, we analyzed TKAs consecutively performed by a single surgeon between January 2019 and August 2021 who had been preoperatively planned to undergo implantation of cruciate-retaining (CR) prostheses. The outcome was whether the PCL was retained successfully. When the PCLs were sacrificed, we used anterior-stabilized (AS) tibial bearings intraoperatively. We retrospectively obtained age, sex, body mass index (BMI), and preoperative diagnosis from the patients' medical records. We measured the medial-lateral width of epicondyle (MLW), the medial posterior condyle height (MPCH), the lateral posterior condyle height (LPCH), the ratio of MLW and MPCH and the ratio of MLW and $\mathrm{LPCH}$, the insall-salvati index, and the severity of the varus or valgus deformity using preoperative radiographs. We fitted univariate and multivariate logistic regression to assess the association of these factors with the successful retention of PCL. We also compared the size of the tibial and femoral components, the thickness of the polyethylene insert, and the replacement of the patella between the CR and AS groups using t tests or chi-square tests to examine the influence of sacrifice of the PCL on the surgical procedure.

Results: Among 307 TKAs, 89 (29.0\%) procedures sacrificed the PCL and converted to AS prostheses intraoperatively. Knees with rheumatoid arthritis $(p<0.01)$, lower insall-salvati index $(p<0.01)$, and more severe varus deformity $(p=0.011)$ were at a higher risk of sacrificing the PCL intraoperatively. No significant differences in age, sex, BMI, MLW, MPCH, LPCH, ratio of MLW and $\mathrm{MPCH}$, ratio of MLW and LPCH, size of the tibial and femoral components, or replacement of the patella were identified between the CR and AS groups. Converting from CR to AS was associated with a thicker polyethylene insert $(p<0.01)$.

Conclusion: Rheumatoid arthritis, lower insall-salvati index, and more severe varus deformity were associated with an increased risk of sacrificing the PCL in TKAs planned to undergo implantation CR prostheses. Converting to AS tibial bearing may result in a thicker polyethylen insert. These factors should be carefully considered for the appropriate selection of prosthesis type.

\section{Introduction}

Posterior cruciate ligament (PCL) retaining (CR) and PCL substituting (PS) prostheses are classical options for total knee arthroplasty (TKA). Considering that sacrificing the PCL opens the flexion gap $2 \mathrm{~mm}$ more than the extension gap,[1] a successful CR TKA requires different surgical techniques from the PS TKA. For example, surgeons performing CR TKA should begin by removing the less distal femur to keep the extension space equally small.[2] Furthermore, many knee systems currently under use do not allow a conversion from CR to PS. Therefore, although CR and PS TKAs showed similar outcomes, from a technical perspective, it is preferable to make the decision to use CR or PS prostheses preoperatively.

Surgeons may refer to intraoperative gap measurements to decide whether to preserve the PCL.[3] The reported rate of intraoperative conversion from CR to PS TKA ranges from $9.9 \%$ to $17 \% .[4,5]$ Nonetheless, even for modern implant systems that allow an intraoperative switch from CR to PS, conversion after bone cutting may compromise surgical efficiency and result in undesirable conditions such as a thicker polyethylene insert or posterior flexion instability. $[6,7]$ Additional efforts must be made to avoid or compensate for these problems. Therefore, it was recommended that surgeons envision the selection between CR and PS in the preoperative plan based on history, physical examination, and imaging and laboratory tests, taking into account both the advantages and disadvantages of implant designs.[8] However, there is a paucity regarding the predictors for the successful preservation of PCL for CR TKA. To fill this 
knowledge gap, we conducted a retrospective study to determine the rate and risk factors for sacrificing PCL in preplanned CR TKAs.

\section{Materials And Methods}

This retrospective review was approved by the ethics committee of the Peking University People's Hospital.

\section{Participants}

We retrospectively included 307 knees from 266 patients who underwent TKA consecutively between January 2019 and August 2021 at Peking University People's Hospital. The diagnoses were osteoarthritis for 297 knees and rheumatoid arthritis for 10 knees. Among them, 254 (82.7\%) were women. The average age at the time of TKA was $67 \pm 7$ years (range, 33-90 years). All knees were preoperatively planned to use a CR knee prosthesis and were implanted into the Biomet Vanguard Complete Knee System by one surgeon (DZ).

\section{Surgical technique}

All TKAs were performed under tourniquet control using a subvastus approach through a midline skin incision. Bone cuts were made using a measured resection technique. The distal femoral cut was made at $6^{\circ}$ valgus angulation using an intramedullary guide. Approximately $7 \mathrm{~mm}$ of bone was removed from the distal femur. An increased amount of bone resection was applied when there was a high flexion contracture $\left(>30^{\circ}\right)$. Rotation of the femoral component was determined with reference to the transepicondylar axis. The size of the femoral implant was determined using the anterior-referencing guide. The proximal tibial cut was made using an extramedullary guide perpendicular to the long axis of the tibia. The tibial posterior slope was usually set to $5^{\circ}$. Medial or lateral soft tissue contracture was manually evaluated carefully and released as needed. The PCL was carefully recessed or the posterior slope of the tibia was increased when flexion tightness was indicated by lift-off or paradoxical rolling forward. In cases of mismatched flexionextension or mediolateral gaps after these efforts, the PCL was sacrificed, and an anterior-stabilized (AS) tibial bearing was used. PCL was recessed in 89 (29.0\%) knees. The Selective patella resurfacing was performed. Components were cemented for all knees.

\section{Measurement methods}

We retrospectively obtained age, sex, body mass index (BMI), preoperative diagnosis, size of the tibial and femoral components, and thickness of the polyethylene insert from patients' medical records. The sizes of the femoral components used in our study were $55,57.5,60,62.5,65,67.5,70$, and 72.5 . The sizes of the tibial components used in our study were $63,67,71,75,79$, and 83 . The medial-lateral lengths of the femoral components for sizes $55,57.5,60$, $62.5,65,67.5,70$ and 72.5 were $59,61,64,66,68,71,73$, and $75 \mathrm{~mm}$, respectively. The thicknesses of the polyethylene insert used in our study were 10,12 , and $14 \mathrm{~mm}$.

We defined the medial-lateral width of epicondyle (MLW) as the length of femoral epicondylar axis on the antero-posterior standing view of knee. We measured the medial posterior condyle height (MPCH), the lateral posterior condyle height (LPCH), and the insall-salvati index using lateral view of knees, and mechanical axis using full limb radiograph (Figure 1). These measurements were performed by an orthopedic surgeon (YW) masked from the patients' information using a picture archiving and communication system. The normality of all radiographs was checked. 


\section{Statistical analysis}

Age was classified into 5 categories $(<60,60-65,65-70,70-75$, and $>75)$. BMI was classified into $<=18.5,18.5-<24.0,24.0-$ $<30.0$, and $>=30.0 \mathrm{~kg} / \mathrm{m}^{2}$. The lower extremity mechanical axis was classified into severe varus deformity $\left(>15^{\circ}\right.$ varus), mild varus deformity (varus between $5^{\circ}$ and $15^{\circ}$ ), neutral position (within $5^{\circ}$ varus or valgus), mild valgus deformity (valgus between $5^{\circ}$ and $15^{\circ}$ ), and severe valgus deformity $\left(>15^{\circ}\right.$ valgus). The insall-salvati index was classified into 4 quartiles. The thicknesses of the polyethylene insert used in our study were divided into two groups (10 mm and >10 $\mathrm{mm}$ ). We calculated the difference between the MLW and the medial-lateral lengths of the femoral components to reflect the femoral coverage.

The outcome in the current study was whether the PCL was retained successfully. Patients were thus divided into CR and AS group. We compared continuous variables, such as MLW, LPCH, the ratio of MLW and MPCH, the ratio of MLW and $\mathrm{LPCH}$, and the difference between the MLW and the medial-lateral width of the femoral components between the CR and AS groups using Student's t test. We fitted the chi-squared test to compare categorical variables, such as age, sex, preoperative diagnosis, lower-extremity mechanical axis, the insall-salvati index, the size of the tibial and femoral components, the thickness of the polyethylene insert, and the resurfacing patella, between the CR and AS groups. Univariate analysis was performed to examine the relationships of risk factors to the successful retention of PCL. Then, a Poisson regression with a robust variance estimate was performed to further examine the association between preoperative clinical and radiograph factors and the successful preservation of the PCL.[9] We also performed tests for linear trends by entering the median value of each category of variables of interest as a continuous variable in the models[10]. A two-sided P value of 0.05 was considered statistically significant. All data were analyzed by IBM ${ }^{\circ}$ SPSS ${ }^{\circ}$ Statistics (Version 22.0) software.

\section{Results}

The mean MLW was $8.37 \pm 0.69 \mathrm{~cm}$ in the CR group and $8.40 \pm 0.74 \mathrm{~cm}$ in the AS group. The mean MPCH was $6.33 \pm 0.58$ $\mathrm{cm}$ in the CR group and $6.28 \pm 0.63 \mathrm{~cm}$ in the AS group. The mean LPCH was $6.30 \pm 0.53 \mathrm{~cm}$ in the CR group and $6.25 \pm 0.58$ $\mathrm{cm}$ in the AS group (Table 1). We did not observe any statistically significant difference in the MLW, MPCH or LPCH between CR and AS group. The mean value of the above parameters according to age and sex are shown in Table 2. There was no significant difference between age groups. Men tended to have larger MLW, MPCH, and LPCH $(p<0.001)$.

Table 1.

Comparison of patients' demographic and clinical factors, and radiographic measurements between retaining and recession of PCL. 


\begin{tabular}{|c|c|c|c|c|c|c|}
\hline & \multirow[t]{2}{*}{ CR group } & \multirow[t]{2}{*}{ AS group } & \multicolumn{2}{|c|}{ univariate analysis } & \multicolumn{2}{|c|}{ multivariate analysis } \\
\hline & & & $\mathrm{T} / \mathrm{F}$ & $P$ value & OR & $P$ value \\
\hline $\mathrm{Age}^{\mathrm{a}}$ & $67 \pm 7$ & $66 \pm 8$ & 5.652 & 0.227 & & \\
\hline$<60$ & $26(11.93 \%)$ & $14(15.73 \%)$ & & & - & - \\
\hline $60-65$ & $47(21.56 \%)$ & $19(21.35 \%)$ & & & 0.838 & 0.542 \\
\hline $65-70$ & 71 (32.57\%) & $24(26.97 \%)$ & & & 0.707 & 0.213 \\
\hline $70-75$ & $35(16.06 \%)$ & $22(24.72 \%)$ & & & 1.094 & 0.742 \\
\hline$>75$ & 39 (17.89\%) & $10(11.24 \%)$ & & & 0.548 & 0.098 \\
\hline Gender $^{a}$ & & & 0.045 & 0.833 & & \\
\hline Male & $37(16.97 \%)$ & $16(17.98 \%)$ & & & - & - \\
\hline Female & $181(83.03 \%)$ & $73(88.76 \%)$ & & & 0.979 & 0.927 \\
\hline $\mathrm{BMI}^{\mathrm{a}}$ & $26.87 \pm 3.56$ & $26.49 \pm 3.76$ & 0.938 & 0.626 & & \\
\hline$<=24.0$ & $44(20.18 \%)$ & $22(24.72 \%)$ & & & - & - \\
\hline $24.0-30.0$ & $136(62.39 \%)$ & $54(60.67 \%)$ & & & 0.770 & 0.217 \\
\hline$>30.0$ & $38(17.43 \%)$ & $13(14.61 \%)$ & & & 0.741 & 0.322 \\
\hline Preoperative diagnosis ${ }^{a}$ & & & & 0.008 & & \\
\hline Osteoarthritis & $215(98.62 \%)$ & $82(92.13 \%)$ & & & - & - \\
\hline Rheumatoid arthritis & $3(1.38 \%)$ & $7(7.87 \%)$ & & & 2.563 & $<0.001^{*}$ \\
\hline$M L W^{b}$ & $8.37 \pm 0.69$ & $8.4 \pm 0.74$ & -0.365 & 0.715 & 1.029 & 0.858 \\
\hline $\mathrm{MPCH}^{\mathrm{b}}$ & $6.33 \pm 0.58$ & $6.28 \pm 0.63$ & 0.586 & 0.558 & 0.895 & 0.549 \\
\hline $\mathrm{LPCH}^{\mathrm{b}}$ & $6.3 \pm 0.53$ & $6.25 \pm 0.58$ & 0.666 & 0.506 & 0.827 & 0.308 \\
\hline The ratio of $\mathrm{MLW}$ and $\mathrm{MPCH}^{\mathrm{b}}$ & $0.76 \pm 0.06$ & $0.75 \pm 0.06$ & 1.117 & 0.265 & 0.245 & 0.369 \\
\hline The ratio of MLW and $L P C H^{b}$ & $0.75 \pm 0.05$ & $0.75 \pm 0.05$ & 1.385 & 0.167 & 0.091 & 0.124 \\
\hline Insall-salvati index ${ }^{b}$ & $1.05 \pm 0.15$ & $0.99 \pm 0.16$ & 16.488 & 0.001 & & \\
\hline $0-1 / 4$ & $41(18.81 \%)$ & $36(40.45 \%)$ & & & - & - \\
\hline $1 / 4-2 / 4$ & $56(25.69 \%)$ & $20(22.47 \%)$ & & & 0.636 & 0.060 \\
\hline $2 / 4-3 / 4$ & $60(27.52 \%)$ & $15(16.85 \%)$ & & & 0.458 & $0.002^{*}$ \\
\hline $3 / 4-1$ & $61(27.98 \%)$ & $18(20.22 \%)$ & & & 0.526 & $0.010^{\star}$ \\
\hline$P$ for trend & & & & & 0.100 & $0.006^{*}$ \\
\hline Lower-extremity mechanical axis ${ }^{b}$ & $-8.13 \pm 7.69$ & $-8.22 \pm 11.53$ & & 0.027 & & \\
\hline$>15^{\circ}$ varus & $25(11.47 \%)$ & $20(22.47 \%)$ & & & 2.058 & $0.012^{*}$ \\
\hline
\end{tabular}




\begin{tabular}{|c|c|c|c|c|c|}
\hline Varus between $5^{\circ}$ to $15^{\circ}$ & $137(62.84 \%)$ & $43(48.31 \%)$ & & 1.028 & 0.917 \\
\hline Neutral position & $42(19.27 \%)$ & $15(16.85 \%)$ & & - & - \\
\hline Valgus between $5^{\circ}$ to $15^{\circ}$ & $10(4.59 \%)$ & $7(7.87 \%)$ & & 1.717 & 0.122 \\
\hline Valgus $>15$ & $4(1.83 \%)$ & $4(4.49 \%)$ & & 1.431 & 0.385 \\
\hline$P$ for trend & & & & 0.993 & 0.606 \\
\hline \multicolumn{6}{|l|}{ The first aider ${ }^{\mathrm{C}}$} \\
\hline A3 & $44(20.18 \%)$ & $33(37.08 \%)$ & $0.008^{*}$ & - & - \\
\hline B1 & $36(16.51 \%)$ & $4(1.83 \%)$ & & 0.222 & $0.001^{*}$ \\
\hline $\mathrm{C} 2$ & $11(5.05 \%)$ & $5(5.62 \%)$ & & 0.477 & 0.078 \\
\hline D0 & $21(9.63 \%)$ & $7(7.87 \%)$ & & 0.569 & 0.123 \\
\hline E4 & $44(20.18 \%)$ & 14(15.73\%) & & 0.447 & $0.003^{*}$ \\
\hline F5 & $62(28.44 \%)$ & $26(29.21 \%)$ & & 0.640 & $0.034^{*}$ \\
\hline
\end{tabular}

a adjusted for age, gender and BMI.

$\mathrm{b}$ adjusted for age, gender, $\mathrm{BMI}$, and preoperative diagnosis.

$\mathrm{C}$ adjusted for age, gender, BMI, preoperative diagnosis, Insall-salvati index, Lower-extremity mechanical axis, The ratio of MLW and $\mathrm{MPCH}$, and The ratio of MLW and $\mathrm{LPCH}$.

$* p<0.05$

Table 2

Radiological measurements of knee geometrics according to age and sex. 


\begin{tabular}{|c|c|c|c|c|c|c|c|c|c|}
\hline & & & MLW & $\mathrm{MPCH}$ & $\mathrm{LPCH}$ & $\begin{array}{l}\text { The ratio } \\
\text { of MLW } \\
\text { and } \\
\mathrm{MPCH}\end{array}$ & $\begin{array}{l}\text { The ratio } \\
\text { of } \mathrm{MLW} \\
\text { and } \mathrm{LPCH}\end{array}$ & $\begin{array}{l}\text { Insal- } \\
\text { salvati } \\
\text { index }\end{array}$ & $\begin{array}{l}\text { Lower- } \\
\text { extremity } \\
\text { mechanical } \\
\text { axis }\end{array}$ \\
\hline \multirow[t]{10}{*}{ Age } & $<60$ & Male & $9.59 \pm 0.93$ & $6.97 \pm 0.22$ & $7.15 \pm 0.37$ & $0.74 \pm 0.08$ & $0.75 \pm 0.06$ & $0.91 \pm 0.13$ & $-5.42 \pm 6.89$ \\
\hline & & Female & $8.26 \pm 0.53$ & $6.24 \pm 0.47$ & $6.2 \pm 0.44$ & $0.76 \pm 0.05$ & $0.75 \pm 0.05$ & $1.04 \pm 0.17$ & $-6.53 \pm 8.68$ \\
\hline & $60-$ & Male & $9.29 \pm 0.49$ & $6.94 \pm 0.44$ & $6.85 \pm 0.39$ & $0.75 \pm 0.05$ & $0.74 \pm 0.05$ & $1 \pm 0.25$ & $-9.63 \pm 4.72$ \\
\hline & & Female & $8.13 \pm 0.53$ & $6.2 \pm 0.55$ & $6.18 \pm 0.45$ & $0.76 \pm 0.05$ & $0.76 \pm 0.05$ & $1.05 \pm 0.15$ & $-9.59 \pm 5.18$ \\
\hline & $65-$ & Male & $9.25 \pm 0.58$ & $6.81 \pm 0.56$ & $6.76 \pm 0.48$ & $0.74 \pm 0.05$ & $0.73 \pm 0.05$ & $1.03 \pm 0.16$ & $-8.22 \pm 8.73$ \\
\hline & & Female & $8.26 \pm 0.57$ & $6.19 \pm 0.50$ & $6.17 \pm 0.49$ & $0.75 \pm 0.06$ & $0.75 \pm 0.05$ & $1.05 \pm 0.15$ & $-6.71 \pm 10.13$ \\
\hline & $\begin{array}{l}70- \\
75\end{array}$ & Male & $9.31 \pm 0.55$ & $7.12 \pm 0.59$ & $7.16 \pm 0.40$ & $076 \pm 0.04$ & $0.77 \pm 0.03$ & $0.95 \pm 0.14$ & $-8.51 \pm 3.08$ \\
\hline & & Female & $8.03 \pm 0.58$ & $6.1 \pm 0.48$ & $6.02 \pm 0.45$ & $0.76 \pm 0.06$ & $0.75 \pm 0.05$ & $1.04 \pm 0.12$ & $-8.34 \pm 12.88$ \\
\hline & $>75$ & Male & $9.19 \pm 0.60$ & $7.07 \pm 0.64$ & $6.83 \pm 0.69$ & $0.75 \pm 0.04$ & $0.74 \pm 0.06$ & $1.03 \pm 0.13$ & $-10.15 \pm 5.09$ \\
\hline & & Female & $8.26 \pm 0.53$ & $6.18 \pm 0.59$ & $6.18 \pm 0.42$ & $0.76 \pm 0.07$ & $0.75 \pm 0.05$ & $1.02 \pm 0.14$ & $-9.61 \pm 8.19$ \\
\hline
\end{tabular}

In univariate analysis, Age, sex, BMI, MLW, MPCH, LPCH, the ratio of MLW and MPCH, or the ratio of MLW and LPCH were not significantly associated with the successful retaining of PCL. A lower insall-salvati index, preoperative diagnosis of rheumatoid arthritis, and a higher severity of varus or valgus deformity were significantly associated with risk of sacrificing the PCL (Table 1). In the multivariate analysis, patients with rheumatoid arthritis $(p<0.01)$, lower insall-salvati index $(p<0.01)$, and serious varus deformity $(p=0.012)$ were at an increased risk of sacrificing the PCL. (Table 1$)$

Sacrificing PCL in TKA was not significantly associated with the size of the tibial and femoral components or the replacement of patella. However, we observed an increased risk of using thicker polyethylene insert in the AS group compared with the CR group $(\mathrm{p}=0.03)$. (Table 3$)$

Table 3

Comparison of implanted components between the retaining and recession of PCL. 


\section{5}

57.5

60

62.5

65

67.5

$70 / 72.5$

The size of the tibial component

63

67

71

75

$79 / 83$

The thickness of the polyethylene insert

10

$12 \& 14$

Patella replacement (yes/no)

Difference between MLW and the medial-lateral lengths of the femoral components

* $\mathrm{p}<0.05$

\section{Discussion}

In this retrospective cohort study, we found that patients with rheumatoid arthritis, a lower insall-salvati index, and a serious varus deformity were at a higher risk of sacrificing the PCL in a preoperatively planned CR TKA. The resection of PCL increased the risk of using a thicker polyethylene insert. To our knowledge, the current study was one of the few studies examining clinical and radiographic predictors of the successful preservation of the PCL in China.

Although Inflammatory arthritis was previously reported to be one of the contraindications for CR TKA,[11] advances in implant design, surgical techniques, and rehabilitation have led to an expansion of the indications for CR TKA. Archibeck et al.[12] and Dennis et al.[13] suggested that CR TKA would yield excellent results in rheumatoid patients with knee arthritis. However, we found that patients with rheumatoid arthritis were more likely to convert from a CRtype prosthesis to a AS-type prosthesis during the surgery. Patients with rheumatoid arthritis may have moderate to severe flexion contracture, severe contracture, or dysfunction of the PCL, which are difficult to achieve gap balancing. Our results were consistent with that by Lombardi et al.[14] who recommended CR-TKA only for patients without severe coronal deformity and flexion contracture and PS-TKA for patients with inflammatory arthritis. 
Patients with a significant coronal deformity typically have some bone erosion and/or condylar dysplasia on the concave side of the deformity, while the opposite, convex side of the joint is affected by tension forces with stretched soft tissues, which may bring difficulty in gap balancing and necessitate resection of the PCL. A cadaveric study showed that there was significantly less change in flexion and extension gap after both medial and lateral releases with retention of the PCL.[15] This finding supported that PS-TKA may be more efficient for proper mediolateral (ML) balancing in knees with severe varus or valgus deformity. Laskin et al.[16] reported that PS-TKA provided superior results for patients with a varus deformity greater than $15^{\circ}$. The authors observed significantly better postoperative alignment, flexion, and residual flexion contracture using a posterior stabilized prosthesis than using a posterior cruciate ligament retaining prosthesis. They also reported better results for a control group that used a PCL retaining prosthesis but did not exhibit preoperative severe deformity. Although Faris et al. [17] and Merrill A et al.[18] reported that a PCL retaining prosthesis could be used as long as proper soft tissue balancing was performed at the time of surgery, they pointed out it was more difficult to correct knee angular deformities due to ligament imbalance and larger deformities intraoperatively, which is consistent with our findings that it was more difficult to reach gap balancing in patients with varus deformities greater than $15^{\circ}$, which resulted in sacrifice of the PCL.

Several reports have emphasized that patellar height is an important factor influencing intraoperative soft tissue balance at high flexion angles, especially in PS TKA[19]. The primary function of the PCL is to resist posterior tibial translation. [20] However, it also acts to maintain a stable joint gap between the femur and tibia beyond $90^{\circ}$ flexion.[1] It has been reported that the PCL and the patellar tendon are almost parallel to the longitudinal axis of the tibia when the knee is flexed $90^{\circ}$.[21] Therefore, the patellar tendon is likely to be an important factor in maintaining the joint gap when the knee is flexed. Gejo et al. [22] reported that patellar tendon strain increased gradually with an increase in knee flexion and that patellar tendon strain at $90^{\circ}$ was correlated with joint gap changes in PS TKA. Hiroshi Sasaki et al.[23] found that patients with higher patellar positions showed significantly larger component gaps than the lower group in high flexion angles $\left(90^{\circ}\right.$ and $\left.135^{\circ}\right)$ in PS TKA. They also found that the patella tendon strain might also be smaller in the higher patella group than in the lower patella group. In this study, we found that patients in the higher patella position group were more likely to preserve the PCL than those in the lower patella position group. This may be because the higher patellar position was related to the smaller patellar tendon strain, which made it easier to reach a suitable gap and resulted in preservation of the PCL. However, in this study, we did not measure patellar tendon strain. More attention needs to be paid to the influence of the patellar position during TKA.

No significant difference was found in the size of the tibial and femoral components. Dae Kyung Bae et al.[4] analyzed the factors affecting the conversion from CR to PS TKA in 920 TKAs and found that the conversion rate from CR- to PStype prostheses was higher in patients with a small femoral component size. They attributed these results to morphologic characteristics of the distal femur and the aspect ratio (anteroposterior/ML ratio) in Asian populations.[24] Asian patients have a small and narrow width of femoral condyles, and the current prostheses do not account for the changes in aspect ratio across the femoral condylar size and that the mediolateral sizing of the contemporary femoral components tends to be too large and thus overhang for smaller knees.[25] Mediolateral overhang can result in irritation of the soft tissue or overstuffing of the joint space and increase the incidence of femoral component downsizing.[26] In this situation, additional distal femoral cutting may be required, and it can induce PCL sacrifice because CR TKA allows less joint line elevation than PS TKA.[27] However, in our study, no significant differences were identified in MLW, MPCH, LPCH, the ratio of $M L W$ and $M P C H$, or the ratio of MLW and $L P C H$, suggesting the PCL may be preserved by careful femoral cutting. The relationship between the femoral component and the preservation of PCL still warrant further investigation.

We found that resection of PCL was associated with an increased risk of using a thicker polyethylene insert than the CR group. This is probably due to the increase in the medial-lateral gap in both the flexion and extension gap after posterior cruciate ligament $(\mathrm{PCL})$ resection.[28] 
Our study had strengths and limitations. First, to our knowledge, our study was one of the first retrospective studies to investigate preoperative predictors of sacrificing the PCL in planned CR TKA. Second, we identified risk factors from clinical information and imaging, and provided data on the morphology of femoral condyle and patellar from a sample of Chinese patients. We acknowledge that this study had several limitations. First, it was a retrospective study from a consecutive series using a single prosthesis. Most of the patients were female with osteoarthritic knees and varus deformities. This female predominance in the distribution of varus deformity is common in Asian populations because of differences in disease demographics among ethnic groups and gender differences in the incidence of bowed legs. Second, the tibial and femoral component size, the final slope of the tibial cut surface, and the decision for conversion to the PS-type prosthesis were determined by the planning and experience of a single surgeon, which may be susceptible to bias. Finally, some clinical and radiographic factors, such as the range of motion, angle of flexion contracture, and posterior tibial slope angle, were not assessed due to a lack of radiographs and information. Further studies that cover these limitations need to be conducted.

\section{Conclusion}

patients with rheumatoid arthritis, a lower insall-salvati index, and a serious varus deformity were at a higher risk of sacrificing the PCL in a preoperatively planned CR TKA. The resection of PCL increased the risk of using a thicker polyethylene insert. These factors should be carefully taken into account for the appropriate selection of prosthesis type preoperatively.

\section{Abbreviations}

PCL: posterior cruciate ligament; CR: cruciate retaining; PS: PCL substituting; TKA: total knee arthroplasty; AS: anterior stabilization; BMl: body mass index; MLW: medial-lateral width of epicondyle; MPCH: medial posterior condyle height; LPCH: lateral posterior condyle height; SD: standard deviation.

\section{Declarations}

\section{Acknowledgments}

We would like to thank DX, RL for participating in the operation.

\section{Consent to participate}

Written informed consent was obtained from all participants according to the Declaration of Helsinki.

\section{Authors' contributions}

DZ and QL designed the study. YW and YL collected and analyzed the data and wrote the first draft of manuscript. JL participated in the data collection, analysis, and interpretation. All authors read and approved the final manuscript.

\section{Funding}

This study was supported by the National Key Research and Development Program of China (No. 2020YFC2004900)

\section{Availability of data and materials}


Data will be available upon request by the corresponding authors.

\section{Ethics approval}

This study was approved by the ethics committee of the Peking University People's Hospital.

\section{Consent for publication}

Not Applicable.

\section{Competing interests}

The authors declare that they have no competing interests.

\section{Author details}

1 Arthritis Clinic \& Research Center, Peking University People’s Hospital, Peking University, Beijing, China.

2 Beijing Huairou Hospital of Beijing Traditional Chinese Medicine, Beijing, China.

3 Arthritis Institute, Peking University, Beijing, China.

\section{References}

1. Kadoya Y, Kobayashi A, Komatsu T, Nakagawa S, Yamano Y. Effects of posterior cruciate ligament resection on the tibiofemoral joint gap. Clinical orthopaedics and related research. 2001;(391):210-217.

2. Sierra RJ, Berry DJ. Surgical technique differences between posterior-substituting and cruciate-retaining total knee arthroplasty. J Arthroplasty. 2008;23(7 Suppl):20-23.

3. Kaneyama R, Otsuka M, Shiratsuchi H, Oinuma K, Miura Y, Tamaki T. Criteria for preserving posterior cruciate ligament depending on intra-operative gap measurement in total knee replacement. Bone \& joint research. 2014;3(4):95-100.

4. Bae DK, Song SJ, Kim KI, Hur D, Lee HH. Intraoperative factors affecting conversion from cruciate retaining to cruciate substituting in total knee arthroplasty. Knee Surg Sports Traumatol Arthrosc. 2016;24(10):3247-3253.

5. In Y, Kim SJ, Kim JM, Woo YK, Choi NY, Kang JW. Agreements between different methods of gap balance estimation in cruciate-retaining total knee arthroplasty. Knee Surg Sports Traumatol Arthrosc. 2009;17(1):60-64.

6. Song SJ, Park CH, Bae DK. What to Know for Selecting Cruciate-Retaining or Posterior-Stabilized Total Knee Arthroplasty. Clinics in orthopedic surgery. 2019;11(2):142-150.

7. Schwab JH, Haidukewych GJ, Hanssen AD, Jacofsky DJ, Pagnano MW. Flexion instability without dislocation after posterior stabilized total knees. Clin Orthop Relat Res. 2005;440:96-100.

8. Tanzer M, Makhdom AM. Preoperative Planning in Primary Total Knee Arthroplasty. J Am Acad Orthop Surg. 2016;24(4):220-230.

9. McNutt LA, Wu C, Xue X, Hafner JP. Estimating the relative risk in cohort studies and clinical trials of common outcomes. American journal of epidemiology. 2003;157(10):940-943. 
10. Park SY, Freedman ND, Haiman CA, Le Marchand L, Wilkens LR, Setiawan VW. Association of Coffee Consumption With Total and Cause-Specific Mortality Among Nonwhite Populations. Annals of internal medicine. 2017;167(4):228235.

11. Laskin RS, O'Flynn HM. The Insall Award. Total knee replacement with posterior cruciate ligament retention in rheumatoid arthritis. Problems and complications. Clinical orthopaedics and related research. 1997;(345):24-28.

12. Archibeck MJ, Berger RA, Barden RM, Jacobs JJ, Sheinkop MB, Rosenberg AG et al. Posterior cruciate ligamentretaining total knee arthroplasty in patients with rheumatoid arthritis. The Journal of bone and joint surgery American volume. 2001;83(8):1231-1236.

13. Dennis DA, Clayton ML, O'Donnell S, Mack RP, Stringer EA. Posterior cruciate condylar total knee arthroplasty. Average 11-year follow-up evaluation. Clinical orthopaedics and related research. 1992;(281):168-176.

14. Lombardi AV, Jr., Berend KR. Posterior cruciate ligament-retaining, posterior stabilized, and varus/valgus posterior stabilized constrained articulations in total knee arthroplasty. Instructional course lectures. 2006;55:419-427.

15. Mihalko WM, Miller C, Krackow KA. Total knee arthroplasty ligament balancing and gap kinematics with posterior cruciate ligament retention and sacrifice. American journal of orthopedics (Belle Mead, NJ). 2000;29(8):610-616.

16. Laskin RS. The Genesis total knee prosthesis: a 10-year followup study. Clinical orthopaedics and related research. 2001;(388):95-102.

17. Faris PM, Herbst SA, Ritter MA, Keating EM. The effect of preoperative knee deformity on the initial results of cruciate-retaining total knee arthroplasty. The Journal of arthroplasty. 1992;7(4):527-530.

18. Ritter MA, Faris GW, Faris PM, Davis KE. Total knee arthroplasty in patients with angular varus or valgus deformities of $>$ or $=20$ degrees. The Journal of arthroplasty. 2004;19(7):862-866.

19. Nishizawa Y, Matsumoto T, Kubo S, Muratsu H, Matsushita T, Oka S et al. The influence of patella height on soft tissue balance in cruciate-retaining and posterior-stabilised total knee arthroplasty. International orthopaedics. 2013;37(3):421-425.

20. Gollehon DL, Torzilli PA, Warren RF. The role of the posterolateral and cruciate ligaments in the stability of the human knee. A biomechanical study. The Journal of bone and joint surgery American volume. 1987;69(2):233-242.

21. Komatsu T, Kadoya Y, Nakagawa S, Yoshida G, Takaoka K. Movement of the posterior cruciate ligament during knee flexion-MRI analysis. Journal of orthopaedic research : official publication of the Orthopaedic Research Society. 2005;23(2):334-339.

22. Gejo R, Morita Y, Matsushita I, Sugimori K, Kimura T. Joint gap changes with patellar tendon strain and patellar position during TKA. Clinical orthopaedics and related research. 2008;466(4):946-951.

23. Sasaki H, Kubo S, Matsumoto T, Muratsu H, Matsushita T, Ishida K et al. The influence of patella height on intraoperative soft tissue balance in posterior-stabilized total knee arthroplasty. Knee surgery, sports traumatology, arthroscopy : official journal of the ESSKA. 2012;20(11):2191-2196.

24. Cheng FB, Ji XF, Lai Y, Feng JC, Zheng WX, Sun YF et al. Three dimensional morphometry of the knee to design the total knee arthroplasty for Chinese population. The Knee. 2009;16(5):341-347.

25. Hitt K, Shurman JR, 2nd, Greene K, McCarthy J, Moskal J, Hoeman T et al. Anthropometric measurements of the human knee: correlation to the sizing of current knee arthroplasty systems. The Journal of bone and joint surgery American volume. 2003;85-A Suppl 4:115-122.

26. Dai Y, Scuderi GR, Penninger C, Bischoff JE, Rosenberg A. Increased shape and size offerings of femoral components improve fit during total knee arthroplasty. Knee surgery, sports traumatology, arthroscopy : official journal of the ESSKA. 2014;22(12):2931-2940.

27. Ji SJ, Zhou YX, Jiang X, Cheng ZY, Wang GZ, Ding H et al. Effect of Joint Line Elevation after Posterior-stabilized and Cruciate-retaining Total Knee Arthroplasty on Clinical Function and Kinematics. Chinese medical journal.

Page $12 / 13$ 
2015;128(21):2866-2872.

28. Park SJ, Seon JK, Park JK, Song EK. Effect of PCL on flexion-extension gaps and femoral component decision in TKA. Orthopedics. 2009;32(10 Suppl):22-25.

\section{Figures}

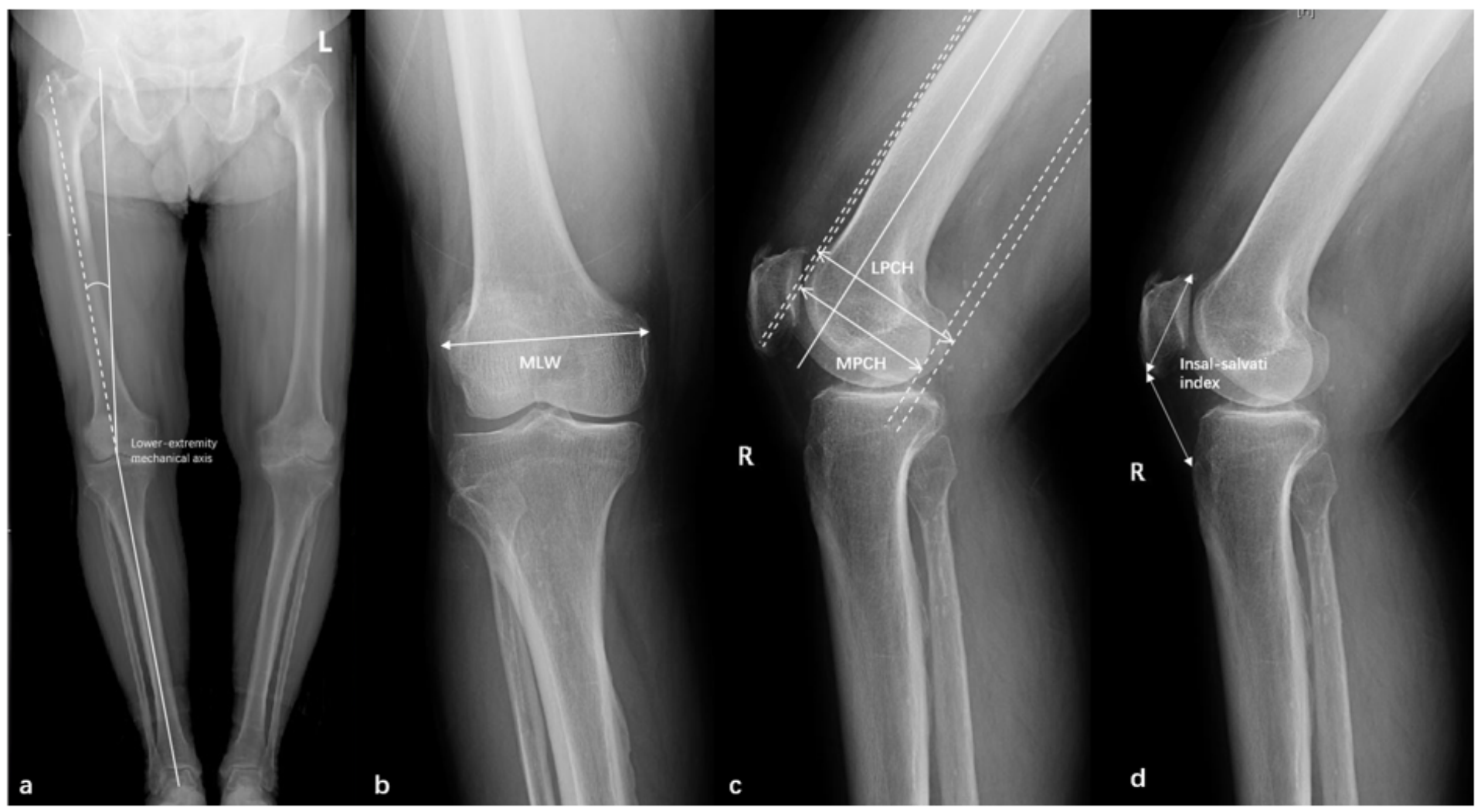

\section{Figure 1}

Measurement of the radiograph factors 